\title{
Pacific
}

Journal of

Mathematics

\section{ASYMPTOTIC EXPANSION AT A CORNER FOR THE}

CAPILLARY PROBLEM: THE SINGULAR CASE

\author{
ERICH MIERSEMANN
}




\title{
ASYMPTOTIC EXPANSION AT A CORNER FOR THE CAPILLARY PROBLEM: THE SINGULAR CASE
}

\section{ERICH MIERSEMANN}

\begin{abstract}
Consider the solution of the capillary surface equation near a corner of the base domain. It is shown that there exists an asymptotic expansion of the height rise of the surface in a wedge when $\alpha+\gamma<\pi / 2$, where $2 \alpha$ is the corner angle and $0 \leq \gamma<\pi / 2$ the contact angle between the surface and the container wall. The asymptotic does not depend on the particular solution considered. In particular, the leading singularity which was discovered by Concus and Finn is equal to the solution up to $O\left(r^{3}\right)$.
\end{abstract}

1. Introduction. We consider the non-parametric capillary problem in the presence of gravity. One seeks a surface $S: u=u(x)$, defined over a base domain $\Omega \subset \mathbb{R}^{2}$, such that $S$ meets vertical cylinder walls over the boundary $\partial \Omega$ in a prescribed constant angle $\gamma$. This problem leads to the equations, see Finn [6],

$$
\operatorname{div} T u=\kappa u \quad \text { in } \Omega,
$$

$$
\nu \cdot T u=\cos \gamma \quad \text { on the smooth parts of } \partial \Omega \text {, }
$$

where

$$
T u=\frac{D u}{\sqrt{1+|D u|^{2}}},
$$

$\kappa=$ const $>0$ and $\nu$ is the exterior unit normal on the smooth parts of $\partial \Omega$.

Let $x=0$ be a corner of $\Omega$ with the interior angle $2 \alpha$ satisfying

$$
0<2 \alpha<\pi \text {. }
$$

We assume that the corner is bounded by lines near $x=0$ and that $\Omega_{R_{0}}=\Omega \cap B_{R_{0}}$ coincides with the circular sector

$$
\left\{x \in \mathbb{R}^{2}\left|x_{1} \tan \alpha \geq\right| x_{2} \mid\right\} \cap B_{R_{0}}
$$

for a sufficiently small $R_{0}>0$, where

$$
B_{R}=\left\{x \in \mathbb{R}^{2} \mid x_{1}^{2}+x_{2}^{2}<R\right\} .
$$

Furthermore, we assume that the contact angle satisfies

$$
0 \leq \gamma<\pi / 2 \text {. }
$$


Concus and Finn [2] have shown that $u$ is bounded near the corner if and only if $\alpha+\gamma \geq \pi / 2$ is satisfied.

The existence of a solution at an isolated corner when $\alpha+\gamma>\pi / 2$ was proved by Emmer [5] and in the case $\alpha+\gamma \leq \pi / 2$ by Finn and Gerhardt [7].

When $\alpha+\gamma>\pi / 2$ there exists an asymptotic expansion of $u$ near the corner, see [9]. In the borderline case $\alpha+\gamma=\pi / 2$ Tam [13] obtained that the normal vector to the surface $S$ is continuous up to the corner.

In this note we will give an asymptotic expansion in the case

$$
\alpha+\gamma<\pi / 2 \text {, }
$$

where the solution $u$ is singular at the corner.

The proof is based on the following comparison principle of Concus and Finn, see [3] or [6, Chapter 5]. This principle remains valid for unbounded domains too, see Finn and Hwang [8].

For $0<R \leq R_{0}$ we set $\Omega_{R}=\Omega \cap B_{R}, \Sigma_{R}=\left(\partial \Omega \cap B_{R}\right) \backslash\{0\}$ and $\Gamma_{R}=\Omega \cap \partial B_{R}$.

THEOREM 1.1 (Concus and Finn [3]). Let $v, w \in C^{2}\left(\Omega_{R} \cup \Gamma_{R}\right)$ with the properties

(i) $\operatorname{div} T v-\kappa v \leq \operatorname{div} T w-\kappa w$ in $\Omega_{R}$,

(ii) $v \geq w$ on $\Gamma_{R}$,

(iii) $\nu \cdot T v \geq \nu \cdot T w$ on $\Sigma_{R}$ as a limit from points of $\Omega_{R}$.

Then $v \leq w$ in $\Omega_{R}$.

This theorem is a consequence of a more general comparison principle, see Finn [6, Chapter 5].

Let $r, \theta$ be polar coordinates centered at $x=0$, set $k=\sin \alpha / \cos \gamma$ and

$$
h(\theta)=\frac{\cos \theta-\sqrt{k^{2}-\sin ^{2} \theta}}{\kappa k} .
$$

Then, it was shown by Concus and Finn [3] that under the assumptions (1.3), (1.4) and (1.5) there exist positive constants $r_{0}$ and $A$ only depending on $\alpha, \gamma, \kappa$ and not on the particular solution $u$ considered such that

$$
\left|u-\frac{h(\theta)}{r}\right| \leq A \quad \text { in } \Omega_{r_{0}}
$$

The author [10] improved this estimate by showing that in fact there exist positive constants $r_{0}, A$ and $\varepsilon$ not dependent on the particular 
solution $u$ considered such that

$$
\left|u-\frac{h(\theta)}{r}\right| \leq A r^{\varepsilon} \quad \text { in } \Omega_{r_{0}} .
$$

The proofs of (1.7) and (1.8) are based on the above comparison principle.

The estimate (1.8) shows that for fixed $\theta$ the height $u(x)$ is asymptotically a hyperbola. For $\theta= \pm \alpha$ one obtains the curves of contact on the container wall. With respect to an experiment performed by Taylor [14] in 1712 compare Finn [6, Note 4, p. 131], see also Boys $[1$, p. 26] and Minkowski [12, p. 577] for the assertion that the trace of the surface on the container wall is a hyperbola.

2. Asymptotic expansion. The proof of the existence of the following expansion is completely based on the comparison principle of Concus and Finn. In contrast to the bounded case $\alpha+\gamma>\pi / 2$, the terms in the expansion do not depend on the particular solution $u$ considered.

In the following we set

$$
h_{-1}(\theta)=h(\theta)
$$

where $h$ is defined by (1.6).

Let $u$ be a solution of (1.1), (1.2) and assume that (1.3), (1.4) and (1.5) hold. Under these assumptions we have

THEOREM 2.1. For a given nonnegative integer $m$ there exist positive constants $r_{0}, A$ and $m+1$ functions $h_{4 l-1}(\theta), l=0, \ldots, m$, analytical on $(-\alpha, \alpha)$ and bounded on $[-\alpha, \alpha]$, such that

$$
\left|u(x)-\sum_{l=0}^{m} h_{4 l-1}(\theta) r^{4 l-1}\right| \leq A r^{4 m+3}
$$

in $\Omega_{r_{0}}$. Moreover, the constants $r_{0}, A$ and the functions $h_{4 l-1}$ do not depend on the particular solution $u$ considered.

REMARK 2.1. The functions $h_{4 l-1}(\theta)$ are solutions of regular boundary value problems of second order (3.16), (3.17) with analytic coefficients and an analytic right-hand side, provided $0<\gamma$ is satisfied, see Lemma 3.2. Therefore, the functions $h_{4 l-1}$ are analytical on the closed interval $[-\alpha, \alpha]$. In the case $\gamma=0$ the boundary value problems are singular, see $\S \S 4$ and 5 .

In particular, we obtain from $m=0$ an improvement of the estimate (1.8). 
Corollary 2.1. Under the assumptions (1.3), (1.4) and (1.5) there exist positive constants $r_{0}$ and $A$ not dependent on the particular solution considered such that

$$
\left|u(x)-\frac{\cos \theta-\sqrt{k^{2}-\sin ^{2} \theta}}{\kappa k r}\right| \leq A r^{3}
$$

in $\Omega_{r_{0}}$.

REMARK 2.2. It is interesting that the above asymptotic expansion has the same qualitative behavior as does the asymptotic expansion for the isolated singular solution of $\operatorname{div} T u=-\kappa u$ studied by Concus and Finn [4]. In particular, both expansions contain only powers in $r^{4 l-1}$ with nonnegative integers $l$.

3. Proof of Theorem 2.1 if $0<\gamma<\pi / 2$. The result follows by induction from the next three lemmas.

For a nonnegative integer $n$ we define

$$
v_{n}(x)=\sum_{l=0}^{n} h_{4 l-1}(\theta) r^{4 l-1}
$$

with analytic functions $h_{4 l-1}(\theta)$ on $[-\alpha, \alpha]$.

We assume that $v_{n}$ satisfies

$$
\begin{array}{ll}
\operatorname{div} T v_{n}-\kappa v_{n}=O\left(r^{4 n+3}\right) & \text { in } \Omega_{R_{0}}, \\
\nu \cdot T v_{n}-\cos \gamma=O\left(r^{4 n+4}\right) & \text { on } \Sigma_{R_{0}} .
\end{array}
$$

By writing $g=O\left(r^{\beta}\right)$, we mean $|g| \leq c r^{\beta}$, where $c$ is independent of $x \in \Omega_{R_{0}}$.

The calculations in the proof of the next lemma are natural extensions of computational results of Concus and Finn [2, 3] and of the author [10].

LEMMA 3.1. Let $v_{n}$ be a function of the above type (3.1) satisfying (3.2) and (3.3). Then there exist positive constants $A$ and $r_{0}, 0<$ $r_{0} \leq R_{0}$, not dependent on the solution $u$ considered such that

$$
\left|u(x)-v_{n}(x)\right| \leq A r^{4 n+3}
$$

in $\Omega_{r_{0}}$.

Proof. Let

$$
w=v+A q(\theta) r^{\lambda}, \quad v=v_{n},
$$


where $A=$ const, $\lambda=$ const $>0$ and $q \in C^{2}[-\alpha, \alpha]$. The positive constant $\lambda$ and the function $q(\theta)$ will be determined later independent of $A$.

In polar coordinates we have

$$
\operatorname{div} T w=r^{-1}\left\{\left(\frac{r w_{r}}{\sqrt{1+|D w|^{2}}}\right)_{r}+\left(\frac{r^{-1} w_{\theta}}{\sqrt{1+|D w|^{2}}}\right)_{\theta}\right\},
$$

where $|D w|^{2}=w_{r}^{2}+r^{-2} w_{\theta}^{2}$.

The definition of $w$ yields

$$
\begin{array}{r}
\operatorname{div} T w=r^{-1}\left\{\left[\frac{r v_{r}+A \lambda q r^{\lambda}}{\left.\sqrt{1+|D v|^{2}}\left(1+\frac{Q}{1+|D v|^{2}}\right)^{-1 / 2}\right]_{r}}\right.\right. \\
\left.+\left[\frac{r^{-1} v_{\theta}+A q^{\prime} r^{\lambda-1}}{\sqrt{1+|D v|^{2}}}\left(1+\frac{Q}{1+|D v|^{2}}\right)^{-1 / 2}\right]_{\theta}\right\}
\end{array}
$$

where

$$
Q=2 A \lambda v_{r} q r^{\lambda-1}+2 A q^{\prime} v_{\theta} r^{\lambda-2}+A^{2} \lambda^{2} q^{2} r^{2 \lambda-2}+A^{2} q^{2} r^{2 \lambda-2} .
$$

We assume that for a given sufficiently small $\eta>0$ the inequality

$$
|A| r_{0}^{\lambda+1} \leq \eta
$$

holds. Then, after some calculations, we obtain

$$
\operatorname{div} T w=\operatorname{div} T v+A L_{\lambda}[q] r^{\lambda}+\eta_{1}+\eta_{2}
$$

in $\Omega_{r_{0}}$, with

$$
L_{\lambda}[q]=\left(a_{1}(\theta) q^{\prime}+a_{0}(\theta) q\right)^{\prime}+b_{1}(\theta) q^{\prime}+b_{0}(\theta) q,
$$

where $a_{1}=h^{2}\left(h^{2}+h^{\prime 2}\right)^{-3 / 2}, \quad a_{0}=\lambda h h^{\prime}\left(h^{2}+h^{\prime 2}\right)^{-3 / 2}, \quad b_{1}=$ $(\lambda+2) h h^{\prime}\left(h^{2}+h^{\prime 2}\right)^{-3 / 2}$ and $b_{0}=\lambda(\lambda+2) h^{\prime 2}\left(h^{2}+h^{\prime 2}\right)^{-3 / 2}$ and the quantities $\eta_{1}$ and $\eta_{2}$ satisfy

$$
\left|\eta_{1}\right| \leq c_{1}|A| r^{\lambda+4}, \quad\left|\eta_{2}\right| \leq c_{2} A^{2} r^{2 \lambda+1}
$$

where the constants $c_{1}$ and $c_{2}$ do not depend on $A$ or $r$.

From (3.4) and (3.6) we obtain

$$
\operatorname{div} T w-\kappa w=A\left(L_{\lambda}[q]-\kappa q\right) r^{\lambda}+\eta_{1}+\eta_{2}+\eta_{3}
$$

in $\Omega_{r_{0}}$, where

$$
\left|\eta_{3}\right| \leq c_{3} r^{4 n+3},
$$

$c_{3}$ independent of $A$ or $r$. 
For $\nu \cdot T w$ on $\Sigma_{R_{0}}$ we have in polar coordinates

$$
\nu \cdot T w=\operatorname{sign} \theta \cdot \frac{r^{-1} w_{\theta}}{\sqrt{1+|D w|^{2}}}, \quad \theta= \pm \alpha .
$$

Under the assumption (3.5) it follows after calculation that

$$
\nu \cdot T w-\cos \gamma=A G_{\lambda}[q] r^{\lambda+1}+\mu_{1}+\mu_{2}+\mu_{3}
$$

on $\Sigma_{r_{0}}$, where

$$
G_{\lambda}[q]=a_{1}(\theta) q^{\prime}+a_{0}(\theta) q
$$

and

$$
\left|\mu_{1}\right| \leq k_{1}|A| r^{\lambda+5}, \quad\left|\mu_{2}\right| \leq k_{2} A^{2} r^{2 \lambda+2}, \quad\left|\mu_{3}\right| \leq k_{3} r^{4 n+4}
$$

with constants $k_{j}$ not dependent on $A$ or $r$.

We insert $q=h(\theta)^{-\lambda}+\varepsilon_{0}$ with $\lambda=4 n+3$ into $L_{\lambda}[q]$ and $G_{\lambda}[q]$, where $\varepsilon_{0}$ is a sufficiently small positive constant such that $L_{\lambda}[q]-\kappa q<0$ remains valid on $[-\alpha, \alpha]$.

If $A>0$, then this choice and (3.8), (3.9) imply that there are positive constants $c_{0}$ and $k_{0}$ not dependent on $r$ or $A$ such that

$$
\operatorname{div} T w-\kappa w \leq-c_{0} A r^{\lambda}+c_{1} A r^{\lambda+4}+c_{2} A^{2} r^{2 \lambda+1}+c_{3} r^{\lambda}
$$

in $\Omega_{r_{0}}$ and

$$
T w-\cos \gamma \geq k_{0} A r^{\lambda+1}-k_{1} A r^{\lambda+5}-k_{2} A^{2} r^{2 \lambda+2}-k_{3} r^{\lambda+1}
$$

on $\Sigma_{r_{0}}$. From (3.5) it follows that

$$
\operatorname{div} T w-\kappa w \leq-r^{\lambda}\left[A\left(c_{0}-c_{1} r_{0}^{4}-c_{2} \eta\right)-c_{3}\right]
$$

in $\Omega_{r_{0}}$, and

$$
\nu \cdot T w-\cos \gamma \geq r^{\lambda+1}\left[A\left(k_{0}-k_{1} r_{0}^{4}-k_{2} \eta\right)-k_{3}\right]
$$

on $\Sigma_{r_{0}}$. The estimate (1.7) or (1.8) implies that for

$$
A=A_{0} r_{0}^{-\lambda}
$$

where $A_{0}$ is a fixed sufficiently large positive constant which does not depend on $r_{0}$ or on the particular solution $u$ considered, we have

$$
u \leq w \quad \text { on } \Gamma_{r_{0}} .
$$


From (3.11), (3.12) and (3.13) we conclude that there exist sufficiently small $\eta>0$ and $r_{0}>0$ such that the estimate (3.5) and the inequalities

$$
\begin{array}{cl}
\operatorname{div} T w-\kappa w \leq 0 & \text { in } \Omega_{r_{0}}, \\
\nu \cdot T w \geq \cos \gamma & \text { on } \Sigma_{r_{0}}, \\
w \geq u & \text { on } \Gamma_{r_{0}}
\end{array}
$$

hold. The comparison principle of Concus and Finn (see Theorem 1.1) implies $u \leq w$ in $\Omega_{r_{0}}$, or

$$
u(x) \leq v_{n}(x)+C r^{4 n+3},
$$

where $C=A h(\theta)^{-(4 n+3)}$.

If $A<0$, then we obtain by the same reasoning a lower bound for $u$ in $\Omega_{r_{0}}$. Thus, the lemma is proved.

The next lemma concerns the existence of approximate solutions in the sense of (3.1), (3.2) and (3.3).

LEMMA 3.2. Assume that $v_{m}(x), m$ a nonnegative integer, satisfies (3.1), (3.2) and (3.3) with $n=m$. Then there exists an analytic function $h_{4 m+3}(\theta)$ on $[-\alpha, \alpha]$ such that

$$
v_{m+1}(x)=v_{m}(x)+h_{4 m+3}(\theta) r^{4 m+3}
$$

satisfies (3.2) and (3.3) with $n=m+1$.

Proof. Set $v=v_{m}$ and $\lambda=4 m+3$. We seek a function $q(\theta)$ such that $w=v(x)+q(\theta) r^{\lambda}$ is an approximate solution in the sense of (3.2) and (3.3) with $n=m+1$.

Following the proof of Lemma 3.1, we obtain

$$
\operatorname{div} T w-\kappa w=\operatorname{div} T v-\kappa v+\left(L_{\lambda}[q]-\kappa q\right) r^{\lambda}+O\left(r^{\lambda+4}\right)
$$

in $\Omega_{r_{0}}$, and

$$
\nu \cdot T w=\nu \cdot T v+G_{\lambda}[q] r^{\lambda+1}+O\left(r^{\lambda+5}\right)
$$

on $\Sigma_{r_{0}}$, provided $r_{0}>0$ is sufficiently small.

Since $v=v_{m}$ is given by (3.1) and satisfies (3.2) and (3.3), we have for an analytic function $f_{\lambda}(\theta)$ on $[-\alpha, \alpha]$

$$
\operatorname{div} T v-\kappa v=f_{\lambda}(\theta) r^{\lambda}+O\left(r^{\lambda+4}\right)
$$


in $\Omega_{r_{0}}$, and for constants $g_{\lambda}^{(1)}, g_{\lambda}^{(2)}$

$$
\nu \cdot T v=\cos \gamma+g_{\lambda}^{(i)} r^{\lambda+1}+O\left(r^{\lambda+5}\right)
$$

on $\Sigma_{r_{0}}^{(i)}$, where $\Sigma^{(1)}$ denotes the upper part and $\Sigma^{(2)}$ the lower part of $\Sigma$.

Thus, we seek a solution $q(\theta)$ of the following linear and regular boundary value problem.

$$
\begin{gathered}
L_{\lambda}[q]-\kappa q=f_{\lambda}(\theta) \\
G_{\lambda}[q]= \begin{cases}g_{\lambda}^{(1)}, & \text { in }(-\alpha, \alpha), \\
g_{\lambda}^{(2)}, & \theta=-\alpha,\end{cases}
\end{gathered}
$$

where the differential operators $L_{\lambda}$ and $G_{\lambda}$ are defined by (3.7) and (3.10).

The lemma is proved if there exists a solution of (3.16) and (3.17).

We will show that the homogeneous problem

$$
\begin{gathered}
L_{\lambda}[q]-\kappa q=0 \quad \text { in }(-\alpha, \alpha), \\
G_{\lambda}[q]=0 \quad \text { if } \theta= \pm \alpha,
\end{gathered}
$$

has only the null solution. Consequently, there is a unique solution of (3.16), (3.17).

Let $q_{0}(\theta)$ be a solution of the homogeneous problem. We replace $v=v_{m}$ by $v_{m}+q_{0}(\theta) r^{\lambda} \ln r$ in $w$ of the proof of Lemma 3.1; see (3.4). Following the calculations in that proof, we obtain

$$
\begin{aligned}
\operatorname{div} T w & =\operatorname{div} T v+A L_{\lambda}[q] r^{\lambda}+\eta_{1}+\eta_{2} \\
& =\operatorname{div} T v_{m}+L_{\lambda}\left[q_{0}\right] r^{\lambda} \ln r+A L_{\lambda}[q] r^{\lambda}+\eta_{1}+\eta_{2}+\eta_{3}
\end{aligned}
$$

where the $\eta_{j}$ satisfy the same estimates as in the proof of Lemma 3.1.

From the definition of $w$ we obtain

$$
\begin{aligned}
\operatorname{div} T w-\kappa w= & \left(L_{\lambda}\left[q_{0}\right]-\kappa q_{0}\right) r^{\lambda} \ln r \\
& +A\left(L_{\lambda}[q]-\kappa q\right) r^{\lambda}+\eta_{1}+\eta_{2}+\eta_{3} .
\end{aligned}
$$

For the boundary operator we find

$$
\begin{aligned}
\nu \cdot T w-\cos \gamma= & G_{\lambda}\left[q_{0}\right] r^{\lambda+1} \ln r+A G_{\lambda}[q] r^{\lambda+1} \\
& +\mu_{1}+\mu_{2}+\mu_{3},
\end{aligned}
$$

also the $\mu_{j}$ satisfy the same estimates as in the proof of Lemma 2.1.

Because $q_{0}$ solves the homogeneous problem (3.18), (3.19), the estimates (3.20) and (3.21) imply by the same reasoning as in the second part of the proof of Lemma 3.1 the asymptotic formula

$$
u(x)=v_{m}(x)+q_{0}(\theta) r^{\lambda} \ln r+O\left(r^{\lambda}\right),
$$

where $\lambda=4 m+3$. 
According to Lemma 2.1, we have on the other hand

$$
u(x)=v_{m}(x)+O\left(r^{\lambda}\right) .
$$

These expansions force that $q_{0}(\theta)=0$ for all $\theta \in[-\alpha, \alpha]$. Thus, the lemma is proved.

REMARK 3.1. The functions $h_{4 l-1}(\theta)$ are even with respect to $\theta=0$ since $h_{-1}(\theta)=h(\theta)$ is even. We omit the details.

REMARK 3.2. The solvability of a boundary value problem of the type (3.16), (3.17) was discussed also in [11] by using asymptotic expansions.

The next lemma, see Finn [6, Chapter 5], which yields the basis of the induction, follows after some calculation.

LEMMA 3.3. The singular function $v_{0}=h(\theta) r^{-1}$ satisfies (3.2) and (3.3), also in the case $\gamma=0$.

4. Proof of Theorem 2.1 if $\gamma=0$. In this case the function $h^{\prime}(\theta), h$ is given by (1.6), becomes singular at the ends of the interval $(-\alpha, \alpha)$ whereas $h$ remains bounded from below by a positive constant.

More precisely, there are positive constants $c_{0}, c_{1}$ and $\varepsilon_{0}, 0<$ $\varepsilon_{0}<\alpha$, such that

$$
\begin{gathered}
c_{0}\left(\alpha^{2}-\theta^{2}\right)^{-1 / 2} \leq h^{\prime}(\theta) \operatorname{sign} \theta \leq c_{1}\left(\alpha^{2}-\theta^{2}\right)^{-1 / 2}, \\
c_{0}\left(\alpha^{2}-\theta^{2}\right)^{-3 / 2} \leq h^{\prime \prime}(\theta) \leq c_{1}\left(\alpha^{2}-\theta^{2}\right)^{-3 / 2}
\end{gathered}
$$

on $\left(-\alpha,-\alpha+\varepsilon_{0}\right) \cup\left(\alpha-\varepsilon_{0}, \alpha\right)$.

Let $v_{n}(x)$ be given by (3.1) with analytic functions $h_{4 l-1}(\theta) \equiv q$ in $(-\alpha, \alpha)$ satisfying

$$
\begin{gathered}
\sup |q(\theta)|<\infty, \\
\sup \left[\left(\alpha^{2}-\theta^{2}\right)^{1 / 2}\left|q^{\prime}(\theta)\right|\right]<\infty, \\
\sup \left[\left(\alpha^{2}-\theta^{2}\right)^{3 / 2}\left|q^{\prime \prime}(\theta)\right|\right]<\infty,
\end{gathered}
$$

where the supremum is taken over $(-\alpha, \alpha)$.

LEMMA 4.1. Let $v_{n}$ be a function of type (3.1), where $h_{4 l-1}$ satisfies (4.3a)-(4.3c) and (3.2). Then the conclusion of Lemma 3.1 is valid. 
Proof. Let $w$ be given by (3.4), where the functions $h_{4 l-1}$ and $q$ satisfy $(4.3 \mathrm{a})-(4.3 \mathrm{c})$. We have

$$
w_{\theta}=r^{-1} h^{\prime}+\sum_{l=1}^{n} h_{4 l-1}^{\prime} r^{4 l-1}+A q^{\prime} r^{\lambda}
$$

and

$$
\begin{aligned}
1+|D w|^{2}=r^{-4}\left(r^{4}+[-h+\right. & \left.\sum_{l=1}^{n}(4 l-1) h_{4 l-1} r^{4 l}+A \lambda q r^{\lambda+1}\right]^{2} \\
& \left.+\left[h^{\prime}+\sum_{l=1}^{n} h_{4 l-1}^{\prime} r^{4 l}+A q^{\prime} r^{\lambda+1}\right]^{2}\right) .
\end{aligned}
$$

Inserting these expressions into

$$
\nu \cdot T w=\operatorname{sign} \theta \cdot \frac{r^{-1} w_{\theta}}{\sqrt{1+|D w|^{2}}},
$$

we obtain from the properties (4.1), (4.3a) and (4.3b) and under the assumption (3.5) that

$$
\nu \cdot T w=1 \quad \text { on } \Sigma_{r_{0}}
$$

as a limit from points of $\Omega_{r_{0}}$.

After some calculation we arrive at (3.8) also in the case $\gamma=0$.

Combining (3.8) and (4.4), Lemma 3.1 follows also in the case $\gamma=0$ as in the second part of the proof of that lemma.

One obtains the estimate (3.8) by the same calculations as in the proof of Lemma 3.1, provided that $\theta \in\left(-\alpha+\varepsilon_{0}, \alpha-\varepsilon_{0}\right)$ is satisfied.

It is not clear from that computation whether the estimates (3.8) remain valid up to the ends of the intervals $(-\alpha, \alpha)$. If $\theta \in$ $\left(-\alpha,-\alpha+\varepsilon_{0}\right] \cup\left[\alpha-\varepsilon_{0}, \alpha\right)$, then we calculate $\operatorname{div} T w$ as follows.

With the abbreviations

$$
\begin{aligned}
& H_{0}=-h(\theta)+\sum_{l=1}^{n}(4 l-1) h_{4 l-1}(\theta) r^{4 l}+A \lambda q(\theta) r^{\lambda+1} \\
& H_{1}=h^{\prime}(\theta)+\sum_{l=1}^{n} h_{4 l-1}^{\prime}(\theta) r^{4 l}+A q^{\prime}(\theta) r^{\lambda+1}
\end{aligned}
$$

we obtain $\operatorname{div} T w=r^{-1}\left(\left[r H_{0}\left(r^{4}+H_{0}^{2}+H_{1}^{2}\right)^{-1 / 2}\right]_{r}+\left[H_{1}\left(r^{4}+H_{0}^{2}+H_{1}^{2}\right)^{-1 / 2}\right]_{\theta}\right)$. 
Since

$$
\begin{aligned}
& {\left[H_{1}\left(r^{4}+H_{0}^{2}+H_{1}^{2}\right)^{-1 / 2}\right]_{\theta}} \\
& \quad=H_{1, \theta}\left(r^{4}+H_{0}^{2}+H_{1}^{2}\right)^{-3 / 2}\left(r^{4}+H_{0}^{2}\right),
\end{aligned}
$$

it follows from (4.1), (4.2) and (4.3a)-(4.3c) that the constants $c_{j}$ in the estimates (3.8) remain bounded near the ends of the interval.

This is valid also for the functions $f_{4 m+3}(\theta)$ in the expansion (3.15).

LEMMA 4.2. Assume that $v_{m}(x), m$ a nonnegative integer, satisfies (3.1), (4.3a)-(4.3c) and (3.2) with $n=m$. Then there exists a function $h_{4 m+3}(\theta)$ satisfying $(4.3 \mathrm{a})-(4.3 \mathrm{c})$ such that the function $v_{m+1}$ from Lemma 3.2 solves the approximate differential equation (3.2) with $n=$ $m+1$.

Proof. As in the proof of Lemma 3.2 we arrive at the differential equation (3.16) for $q=h_{4 m+3}$ with the right-hand side $f_{4 m+3}$.

The boundary conditions are given by $(4.3 \mathrm{a})-(4.3 \mathrm{c})$.

By the same reasoning as in the second part of the proof of Lemma 3.2 one concludes that the associated homogeneous problem has only the solution $q=0$ on $(-\alpha, \alpha)$.

From the lemma of the next section it follows that there is a unique solution of (3.16) under the boundary conditions $(4.3 \mathrm{a})-(4.3 \mathrm{c})$. Thus, Lemma 4.2 is proved.

The result of Theorem 2.1 if $\gamma=0$ follows from the above lemmas and from Lemma 3.3 as in the previous section.

5. The singular boundary value problem. From the behavior (4.1), (4.2) of $h$ one sees that coefficients of the differential equation (3.16) degenerate at the ends of the interval if $\gamma=0$.

LEMMA 5.1. Under the boundary conditions (4.3a)-(4.3c) there exists a unique solution $q \in C^{2}(-\alpha, \alpha)$ of the singular differential equation (3.16) with the right-hand side $f_{\lambda}(\theta)=f_{4 m+3}(\theta)$.

The proof of this lemma is based on standard methods for such singular boundary value problems. For the convenience of the reader we will sketch the proof. by

Let $\phi \in C_{0}^{1}(-\alpha, \alpha)$. We define an associated bilinear form to $L_{\lambda}[q]$

$$
a[q, \phi]=\int_{-\alpha}^{\alpha}\left(a_{1} q^{\prime} \phi^{\prime}+a_{0} q \phi^{\prime}-b_{1} q^{\prime} \phi-b_{0} q \phi+a_{0} q \phi\right) d \theta
$$


Instead of (3.16) we consider

$$
a[q, \phi]+\kappa(q, \phi)=-(f, \phi)
$$

for all $\phi \in C_{0}^{1}(-\alpha, \alpha)$, where $(f, \phi)=\int_{-\alpha}^{\alpha} f(\theta) \phi(\theta) d \theta$.

Define the Hilbert space $\widehat{H}_{1}$ as the closure of $C_{0}^{1}(-\alpha, \alpha)$ with respect to the norm

$$
\|\phi\|_{1}=\left(\int_{-\alpha}^{\alpha} a_{1}(\theta) \phi^{2} d \theta+\|\phi\|_{0}^{2}\right)^{1 / 2},
$$

where $\|\phi\|_{0}^{2}=\int_{-\alpha}^{\alpha} \phi^{2} d \theta$.

From the special structure of the coefficients of $L_{\lambda}[q]$ and the estimate (4.1) for $h^{\prime}(\theta)$ one concludes that there are positive constants $c_{0}$ and $c_{1}$ such that

$$
a[\phi, \phi] \geq c_{1}\|\phi\|_{1}^{2}-c_{0}\|\phi\|_{0}^{2}
$$

for all $\phi \in \widehat{H}_{1}$.

Consequently, according to the lemma of Lax and Milgram, the problem

$$
q \in \widehat{H}_{1}: a[q, \phi]+c(q, \phi)=-(f, \phi)
$$

for all $\phi \in \widehat{H}_{1}$, has a unique solution for each $f \in L^{2}$, provided $c$ is a sufficiently large positive constant.

Since $c(q, \phi)$ is a compact perturbation of $a[q, \phi]+\kappa(q, \phi)$ with respect to the above $\widehat{H}_{1}$ norm, it follows that the associated operator $T$ to $a[q, \phi]+\kappa(q, \phi)$ considered as a mapping from $\widehat{H}_{1}$ in $L^{2}$ has index zero, that means

$$
\text { index } T=\operatorname{codim}(\operatorname{range} T)-\operatorname{dim}(\operatorname{kernel} T)=0 \text {. }
$$

Thus, there is a unique solution of (5.1) for each $f \in L^{2}$, provided the associated homogeneous problem has only the null solution.

The lemma is proved if each solution $q \in \widehat{H}_{1}$ of (5.1) belongs to $C^{2}(-\alpha, \alpha)$ and satisfies $(4.3 \mathrm{a})-(4.3 \mathrm{c})$. The definition of $\widehat{H}_{1}$ implies

$$
\sup \left[\left(\alpha^{2}-\theta^{2}\right)|q(\theta)|\right]<\infty
$$

for $q \in \widehat{H}_{1}$, which is an easy consequence of a Sobolev embedding lemma in one dimension. The supremum is taken over $(-\alpha, \alpha)$. $\operatorname{In}^{3}$ fact, one has the stronger inequality

$$
\sup \left[\left(\alpha^{2}-\theta^{2}\right)^{3 / 4}|q(\theta)|\right]<\infty,
$$

which is a consequence of the Hardy inequality. 
From the special structure of the coefficients of the differential equation (3.16) and since the right-hand side $f$ is bounded on $(-\alpha, \alpha)$, we obtain the desired estimates (4.3a)-(4.3c) after three iterations by using the equation (3.16) and the estimate (5.2). With (5.3) instead of (5.2) we need only two iterations.

Acknowledgment. I thank the referee for some suggestions which improved the presentation of this paper and for calling to my attention some additional references.

\section{REFERENCES}

[1] C. V. Boys, Soap Bubbles, Dover Publications, New York, 1959.

[2] P. Concus and R. Finn, On a class of capillary surfaces, J. Analyse Math., 23 (1970), 65-70.

[3] _ On capillary free surfaces in a gravitational field, Acta Math., 132 (1974), 207-223.

[4] _ $\quad$ A singular solution of the capillary equation, Invent. Math., 29 (1975), 143-148.

[5] M. Emmer, Esistenza, unicità e regolarità nelle superfici di equilibrio nei capillari, Ann. Univ. Ferrara Sez. VII, 18 (1973), 79-84.

[6] R. Finn, Equilibrium Capillary Surfaces, Grundlehren der Mathem. Wiss. 284, Springer-Verlag, New York, 1986. Russian Translation, with Appendix by H. C. Wente, Mir Publisher, Moscow, 1988.

[7] R. Finn and G. Gerhardt, The internal sphere condition and the capillary problem, Ann. Mat. Pura Appl., 112 (1977), 13-31.

[8] R. Finn and J.-F. Hwang, On the comparison principle for capillary surfaces, J. Fac. Sci. Univ. Tokyo, 36 (1989), 131-134.

[9] E. Miersemann, Asymptotic expansion at a corner for the capillary problem, Pacific J. Math., 134 (1988), 299-311.

[10] On the behaviour of capillaries at a corner, Pacific J. Math., 140 (1989), 149-153.

[11] _ On the singular behaviour of fluid in vertical wedge, Z. Anal. Anw., 9 (1990), 467-471.

[12] H. Minkowski, Kapillarität, Encyklopädie der Mathematischen Wissenschaften, Physik Band 5.1, 558-613, Teubner-Verlag Leipzig, 1903-1921.

[13] L.-F. Tam, Regularity of capillary surfaces over domains with corners: borderline case, Pacific J. Math., 125 (1986), 469-485.

[14] B. Taylor, Concerning the ascent of water between two glass planes, Phil. Trans. Roy. Soc. London, 27 (1712), 538.

Received December 15, 1990.

UNIVERSITÄT LEIPZIG

0-7010 LEIPZIG, GERMANY 



\title{
PACIFIC JOURNAL OF MATHEMATICS
}

Founded by

\author{
E. F. Beckenbach (1906-1982) F. Wolf (1904-1989)
}

\section{EDITORS}

V. S. VARADARAJAN

(Managing Editor)

University of California

Los Angeles, CA 90024-1555

vsv@math.ucla.edu

Herbert Clemens

University of Utah

Salt Lake City, UT 84112

clemens@math.utah.edu

F. Michael Christ

University of California

Los Angeles, CA 90024-1555

christ@math.ucla.edu

THOMAS ENRIGHT

University of California, San Diego

La Jolla, CA 92093

tenright@ucsd.edu
Nicholas ERcolani

University of Arizona

Tucson, AZ 85721

ercolani@math.arizona.edu

R. FInN

Stanford University

Stanford, CA 94305

finn@gauss.stanford.edu

VAughan F. R. Jones

University of California

Berkeley, CA 94720

vfr@math.berkeley.edu

STEVEN KERCKHOFF

Stanford University

Stanford, CA 94305

spk@gauss.stanford.edu

\section{C. MOORE}

University of California

Berkeley, CA 94720

MARTIN SCHARLEMANN

University of California

Santa Barbara, CA 93106

mgscharl@henri.ucsb.edu

Harold Stark

University of California, San Diego La Jolla, CA 92093

\section{SUPPORTING INSTITUTIONS}

UNIVERSITY OF ARIZONA

UNIVERSITY OF BRITISH COLUMBIA

CALIFORNIA INSTITUTE OF TECHNOLOGY

UNIVERSITY OF CALIFORNIA

MONTANA STATE UNIVERSITY

UNIVERSITY OF NEVADA, RENO

NEW MEXICO STATE UNIVERSITY

OREGON STATE UNIVERSITY
UNIVERSITY OF OREGON

UNIVERSITY OF SOUTHERN CALIFORNIA

STANFORD UNIVERSITY

UNIVERSITY OF HAWAII

UNIVERSITY OF TOKYO

UNIVERSITY OF UTAH

WASHINGTON STATE UNIVERSITY

UNIVERSITY OF WASHINGTON 


\section{PACIFIC JOURNAL OF MATHEMATICS}

Volume $157 \quad$ No. $1 \quad$ January 1993

Permutation enumeration symmetric functions, and unimodality

FRANCESCO BRENTI

On the analytic reflection of a minimal surface

JAIGYOUNG CHOE

Contractive zero-divisors in Bergman spaces

Peter Larkin DUREn, DMitry Khavinson, Harold SEymour

SHAPIRO and CARL SUNDBERG

On the ideal structure of positive, eventually compact linear operators on

Banach lattices

RUEY-JEN JANG and HAROLD DEAN VICTORY, JR.

A note on the set of periods for Klein bottle maps

JAUME LLIBRE

Asymptotic expansion at a corner for the capillary problem: the singular case

\section{ERICH MIERSEMANN}

A state model for the multivariable Alexander polynomial

JUN MURAKAMI

Free Banach-Lie algebras, couniversal Banach-Lie groups, and more

Vladimir G. Pestov

Four manifold topology and groups of polynomial growth

RICHARD ANDREW STONG

A remark on Leray's inequality

AKIRA TAKESHITA

$A_{\infty}$ and the Green function

JANG-MEI GLORIA WU

Integral spinor norms in dyadic local fields. I 